\title{
Stochasticity in radiative polarization of ultrarelativistic electrons in an ultrastrong laser pulse
}

\author{
Ren-Tong Guo, ${ }^{1,2, *}$ Yu Wang,,${ }^{1,2, *}$ Rashid Shaisultanov, ${ }^{2}$ Feng Wan, ${ }^{1}$ Zhong-Feng Xu, ${ }^{1}$ Yue-Yue Chen, ${ }^{3,2, \dagger}$ \\ Karen Z. Hatsagortsyan ${ }^{2},{ }^{2, *}$ and Jian-Xing Li ${ }^{1,2, \S}$ \\ ${ }^{1}$ MOE Key Laboratory for Nonequilibrium Synthesis and Modulation of Condensed Matter, School of Physics, \\ Xi'an Jiaotong University, Xi'an 710049, China \\ ${ }^{2}$ Max-Planck-Institut für Kernphysik, Saupfercheckweg 1, 69117 Heidelberg, Germany \\ ${ }^{3}$ Department of Physics, Shanghai Normal University, Shanghai 200234, China
}

(Received 25 March 2020; accepted 1 September 2020; published 24 September 2020)

\begin{abstract}
Stochastic effects in the spin (de)polarization of an ultrarelativistic electron beam during photon emissions in a counterpropagating ultrastrong focused laser pulse in the quantum radiation reaction regime are investigated. We employ a Monte Carlo method to describe the electron dynamics semiclassically and photon emission and electron radiative polarization quantum mechanically. While in the latter the photon emission is inherently stochastic, we are able to identify its imprints in comparison with the semiclassical stochasticity-free method of radiative polarization applicable in the quantum regime. With an initially-spin-polarized electron beam, the impact of stochastic effects of photon emissions on the spin observable is demonstrated in the dependence of the depolarization degree on the electron scattering angle and the final electron energy (spin stochastic diffusion). With an initially unpolarized electron beam, the stochastic effects on the spin are exhibited in enhancing the known effect of splitting of the electron beam along the propagation direction into two oppositely polarized parts by an elliptically polarized laser pulse. The considered stochastic effects for the spin are observable with currently achievable laser and electron-beam parameters.
\end{abstract}

DOI: 10.1103/PhysRevResearch.2.033483

\section{INTRODUCTION}

The modern laser technique is advancing rapidly, and the state-of-the-art ultrastrong ultrashort laser pulses can achieve peak intensities of about $10^{22} \mathrm{~W} / \mathrm{cm}^{2}$, with a duration of about tens of femtoseconds and an energy fluctuation on the order of 1\% [1-7]. In such strong fields QED processes become nonlinear [8]. The large classical nonlinearity parameter in strong fields, which assumes that the electron energy gain in the laser field over the Compton wavelength is larger than the laser photon energy, gives access to multiphoton QED processes. Furthermore, the large quantum nonlinearity parameter in strong fields, which assumes that the laser field in the electron rest frame is comparable to the QED critical field, allows for the large quantum recoil in photon emissions and sizable probability for electron-positron pair production. Thus, the strong laser fields open an avenue for investigation of nonlinear QED processes, beginning with the famous SLAC E-144 experiment $[9,10]$ and recently realized in all optical setups [11-13]. The fundamental processes of nonlinear QED are nonlinear Compton scattering, multiphoton

\footnotetext{
*These authors have contributed equally to this work.

†yueyuechen@shnu.edu.cn

¥k.hatsagortsyan@mpi-hd.mpg.de

§jianxing@xjtu.edu.cn
}

Published by the American Physical Society under the terms of the Creative Commons Attribution 4.0 International license. Further distribution of this work must maintain attribution to the author(s) and the published article's title, journal citation, and DOI.
Breit-Wheeler (BW) processes, and nonlinear Bethe-Heitler $(\mathrm{BH})$ processes. In nonlinear Compton scattering an electron can absorb millions of laser photons to emit a high-energy $\gamma$ photon [14-16]. In the multiphoton BW process a $\gamma$ photon interacting with the laser fields generates an electron-positron pair [17]. In the nonlinear $\mathrm{BH}$ process an electron-positron pair is created in the interaction of a highly intense laser field with a nuclear Coulomb field [18]. There are far-reaching plans to investigate nonlinear QED processes during ultrastrong laser-plasma interactions [19].

Radiation reaction effects were discovered long ago in classical electrodynamics [20-22], as well as in the quantum domain [18], and recently have attracted wide attention due to the possibility of observing those effects directly in radiative processes in laser fields [23]. Thus, recently, classical and quantum signatures of the radiation reaction in electron energy losses have been identified in the experiments of an ultrarelativistic electron-beam collision with strong laser fields [24,25]. Quantum features of the radiation reaction originate from the discrete and probabilistic character of a photon emission, which gives rise to stochasticity effects. The latter is responsible for broadening of the energy spread of an electron beam in a plane laser field [26-28], causes electron stochastic heating in a standing laser field [29], results in quantum quenching of radiation losses in short laser pulses [30], disturbs the angular distribution of radiation [31,32], and brings about the so-called electron straggling effect $[33,34]$, when the electron propagates a long distance without radiation, resulting in an increase of the yield of high-energy photons.

Polarization of electrons and photons adds another dimension to the investigation of nonlinear QED and radiation reaction processes in laser fields. Nonlinear QED processes 
with polarized electrons and photons in the initial and final states have been studied less, but offer a more promising direction of investigation. In particular, the polarized highenergy $\gamma$-ray interaction with a quantum vacuum promises an enhanced signal for the coveted detection of vacuum birefringence [35-39]. Since the cross section of nonlinear Compton scattering is electron spin and photon polarization dependent [40], highly polarized $\gamma$ rays can be generated by initially spin-polarized electrons via nonlinear Compton scattering [41]. Similarly, the cross section of nonlinear BW pair production relies on photon polarization [42] and consequently the intermediate photon polarization should be involved in simulations of nonlinear BW pair production in laser and electron-beam collisions $[43,44]$.

The radiation reaction can have an impact on the electron spin dynamics, proved long ago for synchrotron radiation. It may induce polarization of the unpolarized electron beams (the Sokolov-Ternov effect) [45-48] or depolarization of the initially polarized beam [49,50]. Recently, there have been several proposals on how to use ultrastrong laser fields for generation of polarized relativistic electron beams [51-59]. Polarized electrons are commonly generated by accelerating nonrelativistic polarized electrons, obtained from photocathodes [60], spin filters [61], and beam splitters [62], by conventional accelerators [63] and laser wake-field accelerators $[64,65]$, or by radiative polarization in storage rings [66,67], in which the polarization typically requires a period from minutes to hours because of the modest magnetic fields in storage rings on the order of tesla. In contrast, laser beams have a potential to polarize electrons within tens of femtoseconds. In particular, the possibilities for creation of ultrarelativistic high-polarization high-density electron and positron beams in femtoseconds via utilizing an asymmetric spin-dependent radiation reaction in elliptically polarized laser fields are shown in [54-56] and using two-color laser fields in [57-59]. While polarization-resolved probabilities for nonlinear QED processes in a monochromatic plane-wave laser field have been calculated in Refs. $[68,69]$, they yield cumbersome expressions for the probabilities, which are impractical to use in Monte Carlo and particle-in-cell simulations. More simple formulas for probabilities of polarization-resolved processes have been advanced in Refs. [54,55] using local constant field approximation and developing appropriate stochastic algorithms to treat polarization-resolved nonlinear QED processes in ultrastrong laser-electron-beam interactions. The methods put forward in these works create an opportunity for detailed investigation of all features of the radiative polarization and depolarization processes in ultrastrong focused laser fields, as well as in multiple laser-beam configurations. Usually, a full quantum mechanical study of the radiation reaction includes all quantum effects, such as the photon recoil, stochasticity, and interferences, which makes it difficult to single out the specific radiation reaction signatures of the stochasticity.

In this work, the impact of stochastic effects due to discrete photon emissions on the radiative (de)polarization of an ultrarelativistic electron-beam colliding head-on with an ultrastrong laser pulse is investigated in the quantum radiation reaction regime (see the interaction scenarios in Fig. 1). We employ a Monte Carlo (MC) method to describe spin-resolved electron dynamics in a strong laser field,

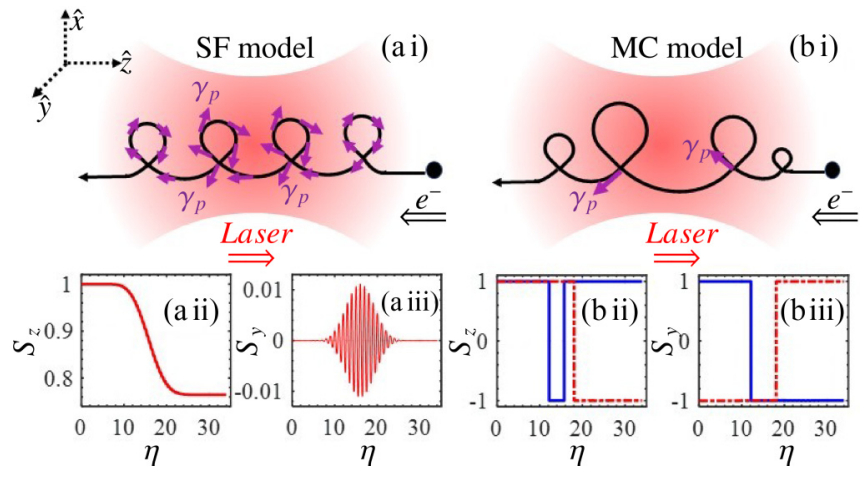

FIG. 1. Scenario for the detection of stochasticity effects in radiative depolarization. The trajectories and spin evolutions of LSP electrons colliding head-on with a linearly polarized laser pulse, polarizing in the $x$ direction and propagating in the $z$ direction in (a) the SF model and (b) the MC model. Here $S_{\alpha}$ is the spin component measured along the $\alpha$ axis, $\alpha=\{x, y, z\}$, and $e^{-}$and $\gamma_{p}$ indicate the electron and emitted photon, respectively. Red dash-dotted and blue solid curves in (b ii) and (b iii) show two different sample electrons, respectively, and $\eta$ the laser phase.

stochastic photon emissions, and corresponding stochastic radiative spin evolution. To elucidate the role of stochastic spin effects, we develop an auxiliary semiclassical stochasticity-free (SF) method for the description of the spindependent radiation reaction in electron dynamics. For this purpose we use the Baier-Katkov-Strakhovenko equation for the expectation value of the electron spin $[48,70]$, which is a generalization of the Thomas-Bargmann-Michel-Telegdi (TBMT) equation [71-73], including the radiation reaction for the electron spin. The latter is supplemented with the modified Landau-Lifshitz equation [54], including the spindependent radiation reaction and the quantum recoil. We consider a depolarization scenario for the initially longitudinally spin-polarized (LSP) (with velocity, along the $z$ axis) electron beam. The depolarization proceeds in different ways in semiclassical and quantum models, which after the interaction yields differences in the angle-resolved polarization distribution of the electron beam and provides the signatures of photon emission stochasticity in the spin radiative dynamics. In particular, in the SF model an electron continuously loses energy due to radiation (without stochastic effects and straggling in photon emissions, the photon energies are typically low), which gradually alters the electron trajectory and the spin longitudinal component due to the radiation reaction, while the spin component along the laser magnetic field oscillates in the symmetric laser field, as shown in Figs. 1(a i)-1(a iii). In contrast, in the MC model a finite number of photons are stochastically emitted with random energies and discretely alter the electron dynamics due to the quantum recoil, and the quantum spin state stochastically flips on the instantaneous spin quantization axis (SQA) [see Figs. $1(\mathrm{~b}$ i $)-1(\mathrm{~b}$ iii)]. The signature characteristics of the stochasticity are identified by analyzing the features of the stochastic spin diffusion. In the second applied scenario, we use an initially unpolarized electron beam colliding head-on with an elliptically polarized laser pulse. Here the unpolarized electron beam splits along the propagation direction into 
two oppositely polarized parts, and the stochasticity effect is observed in enhancing the separation.

\section{SIMULATION METHODS}

In this work we employ ultrastrong laser fields with an invariant field parameter $a_{0} \equiv e E_{0} / m \omega_{0} \gg 1[8,40]$, where $E_{0}$ and $\omega_{0}$ are the laser field amplitude and frequency, respectively, and $-e$ and $m$ are the electron charge and mass, respectively. Relativistic units with $c=\hbar=1$ are used throughout. The quantum radiation reaction regime requires the invariant quantum parameter $\chi \equiv|e| \sqrt{-\left(F_{\mu \nu} p^{v}\right)^{2}} / m^{3} \gtrsim 1$ $[8,40]$, with the field tensor $F_{\mu \nu}$ and the four-vector of the electron momentum $p^{\nu}$. In the electron-laser counterpropagating scheme, $\chi \approx 2 a_{0} \gamma_{e} \omega_{0} / m$, with the electron Lorentz factor $\gamma_{e}$.

\section{A. The MC method}

In the MC method, we treat spin-resolved electron dynamics semiclassically and photon emissions quantum mechanically in the local constant field approximation [8,40,74,75], valid at $a_{0} \gg 1$. At each simulation step, given the smallness of the emission angle on the order of $1 / \gamma$ for an ultrarelativistic electron, the photon emission is assumed to be with the electron velocity and the photon emission is determined by the angle-integrated probabilities [41] (for moderate-energy photons the inclusion of the angle for photon emission could affect the radiation beaming [76]), derived in the Baier-Katkov QED operator method [42]

$$
\frac{d^{2} W_{f i}}{d u d \eta}=\frac{W_{R}}{2}\left(F_{0}+\xi_{1} F_{1}+\xi_{2} F_{2}+\xi_{3} F_{3}\right),
$$

where

$$
\begin{aligned}
F_{0}= & -(2+u)^{2}\left[\operatorname{Int} K_{1 / 3}\left(u^{\prime}\right)-2 K_{2 / 3}\left(u^{\prime}\right)\right]\left(1+\mathbf{S}_{i f}\right) \\
& +u^{2}\left(1-\mathbf{S}_{i f}\right)\left[\operatorname{Int} K_{1 / 3}\left(u^{\prime}\right)+2 K_{2 / 3}\left(u^{\prime}\right)\right] \\
& +2 u^{2} \mathbf{S}_{i f} \operatorname{Int} K_{1 / 3}\left(u^{\prime}\right)-\left(4 u+2 u^{2}\right)\left(\mathbf{S}_{f}+\mathbf{S}_{i}\right)[\hat{\mathbf{v}} \\
& \times \hat{\mathbf{a}}] K_{1 / 3}\left(u^{\prime}\right)-2 u^{2}\left(\mathbf{S}_{f}-\mathbf{S}_{i}\right)[\hat{\mathbf{v}} \times \hat{\mathbf{a}}] K_{1 / 3}\left(u^{\prime}\right) \\
& -4 u^{2}\left[\operatorname{Int} K_{1 / 3}\left(u^{\prime}\right)-K_{2 / 3}\left(u^{\prime}\right)\right]\left(\mathbf{S}_{i} \cdot \hat{\mathbf{v}}\right)\left(\mathbf{S}_{f} \cdot \hat{\mathbf{v}}\right) ; \\
F_{1}=- & 2 u^{2} \operatorname{Int} K_{1 / 3}\left(u^{\prime}\right)\left\{\left(\mathbf{S}_{i} \hat{\mathbf{a}}\right) \mathbf{S}_{f}[\hat{\mathbf{v}} \times \hat{\mathbf{a}}]+\left(\mathbf{S}_{f} \hat{\mathbf{a}}\right) \mathbf{S}_{i}[\hat{\mathbf{v}} \times \hat{\mathbf{a}}]\right\} \\
+ & 4 u\left[\left(\mathbf{S}_{i} \cdot \hat{\mathbf{a}}\right)(1+u)+\left(\mathbf{S}_{f} \cdot \hat{\mathbf{a}}\right)\right] K_{1 / 3}\left(u^{\prime}\right) \\
+ & 2 u(2+u) \hat{\mathbf{v}}\left[\mathbf{S}_{f} \times \mathbf{S}_{i}\right] K_{2 / 3}\left(u^{\prime}\right) ; \\
F_{2}=- & \left(2 u^{2}\left\{\left(\mathbf{S}_{i} \hat{\mathbf{v}}\right) \mathbf{S}_{f}[\hat{\mathbf{v}} \times \hat{\mathbf{a}}]+\left(\mathbf{S}_{f} \hat{\mathbf{v}}\right) \mathbf{S}_{i}[\hat{\mathbf{v}} \times \hat{\mathbf{a}}]\right\}+2 u(2+u)\right. \\
\times & \left.\hat{\mathbf{a}}\left[\mathbf{S}_{f} \times \mathbf{S}_{i}\right]\right) K_{1 / 3}\left(u^{\prime}\right)-4 u\left[\left(\mathbf{S}_{i} \cdot \hat{\mathbf{v}}\right)+\left(\mathbf{S}_{f} \cdot \hat{\mathbf{v}}\right)(1+u)\right] \\
\times & \operatorname{Int} K_{1 / 3}\left(u^{\prime}\right)+4 u(2+u)\left[\left(\mathbf{S}_{i} \cdot \hat{\mathbf{v}}\right)+\left(\mathbf{S}_{f} \cdot \hat{\mathbf{v}}\right)\right] K_{2 / 3}\left(u^{\prime}\right) ;
\end{aligned}
$$

$$
\begin{aligned}
F_{3}= & 4\left[1+u+\left(1+u+\frac{u^{2}}{2}\right) \mathbf{S}_{i f}-\frac{u^{2}}{2}\left(\mathbf{S}_{i} \cdot \hat{\mathbf{v}}\right)\left(\mathbf{S}_{f} \cdot \hat{\mathbf{v}}\right)\right] K_{2 / 3}\left(u^{\prime}\right) \\
& +2 u^{2}\left\{\mathbf{S}_{i}[\hat{\mathbf{v}} \times \hat{\mathbf{a}}] \mathbf{S}_{f}[\hat{\mathbf{v}} \times \hat{\mathbf{a}}]-\left(\mathbf{S}_{i} \cdot \hat{\mathbf{a}}\right)\left(\mathbf{S}_{f} \cdot \hat{\mathbf{a}}\right)\right\} \operatorname{Int} K_{1 / 3}\left(u^{\prime}\right) \\
& -4 u\left\{(1+u) \mathbf{S}_{i}[\hat{\mathbf{v}} \times \hat{\mathbf{a}}]+\mathbf{S}_{f}[\hat{\mathbf{v}} \times \hat{\mathbf{a}}]\right\} K_{1 / 3}\left(u^{\prime}\right) ;
\end{aligned}
$$

$W_{R}=\alpha m /\left[8 \sqrt{3} \pi \lambda_{c}\left(k \cdot p_{i}\right)(1+u)^{3}\right] ; u=\varepsilon_{\gamma} /\left(\varepsilon_{i}-\varepsilon_{\gamma}\right) ; u^{\prime}=$ $2 u / 3 \chi ; \quad \operatorname{Int} K_{1 / 3}\left(u^{\prime}\right) \equiv \int_{u^{\prime}}^{\infty} d z K_{1 / 3}(z) ; K_{n}$ is the $n$ th-order modified Bessel function of the second kind; $\alpha$ is the finestructure constant; $\lambda_{c}$ is the Compton wavelength; $\varepsilon_{\gamma}$ is the emitted photon energy; $\varepsilon_{i}$ is the electron energy before radiation; $\eta=k \cdot r$ is the laser phase; $p_{i}, k$, and $r$ are four-vectors of the electron momentum before radiation, the laser wave vector, and the coordinate, respectively; $\mathbf{S}_{i}$ and $\mathbf{S}_{f}$ are the electron spin-polarization vector before and after radiation, respectively; $\left|\mathbf{S}_{i, f}\right|=1$; and $\mathbf{S}_{i f} \equiv \mathbf{S}_{i} \cdot \mathbf{S}_{f}$. The photon polarization is represented by the Stokes parameters $\xi_{1}, \xi_{2}$, and $\xi_{3}$, defined with respect to the axes $\hat{\mathbf{e}}_{1}=\hat{\mathbf{a}}-\hat{\mathbf{v}}(\hat{\mathbf{v}} \hat{\mathbf{a}})$ and $\hat{\mathbf{e}}_{2}=$ $\hat{\mathbf{v}} \times \hat{\mathbf{a}}$ [77], with the photon emission direction $\hat{\mathbf{n}}$ along the electron velocity $\mathbf{v}$ for the ultrarelativistic electron, $\hat{\mathbf{v}}=\mathbf{v} /|\mathbf{v}|$, and the unit vector $\hat{\mathbf{a}}=\mathbf{a} /|\mathbf{a}|$ along the electron acceleration a. As the radiation probability in Eq. (1) sums over the photon polarization and the electron final spin after radiation $\bar{W}_{f i} \geqslant$ $N_{r}$, a photon is emitted, and the emitted photon energy $\omega_{\gamma}$ is determined by the condition $\frac{1}{\bar{W}_{f i}} \int_{\omega_{0}}^{\omega_{\gamma}} \frac{d \bar{W}_{f i}(\omega)}{d \omega} d \omega=N_{r}^{\prime}$ [78-80], where $N_{r}$ and $N_{r}^{\prime}$ are two random numbers in [0,1]. We choose two orthogonal pure states of the Stokes parameters $\hat{\xi}^{ \pm} \equiv$ $\pm\left(\xi_{1}^{\text {mix }}, \xi_{2}^{\text {mix }}, \xi_{3}^{\text {mix }}\right) / \xi_{0}^{\text {mix }}$, with $\xi_{1}^{\text {mix }}=F_{1} / F_{0}, \quad \xi_{2}^{\text {mix }}=F_{2} / F_{0}$, $\xi_{3}^{\text {mix }}=F_{3} / F_{0}$, and $\xi_{0}^{\text {mix }} \equiv \sqrt{\left(\xi_{1}^{\text {mix }}\right)^{2}+\left(\xi_{2}^{\text {mix }}\right)^{2}+\left(\xi_{3}^{\text {mix }}\right)^{2}}$, and the corresponding probabilities of the photon emission in these two states $W_{f i}^{ \pm}$can be obtained by Eq. (1). If $W_{f i}^{+} / \bar{W}_{f i} \geqslant$ $N_{r}^{\prime \prime}$ the $\hat{\xi}^{+}$photon state is chosen; otherwise the photon state is set to $\hat{\xi}^{-}$, with a random number $N_{r}^{\prime \prime} \in[0,1]$. For the photon observation, the Stokes parameters of each emitted photon should be rotated from the instantaneous frame to the observation frame (for more details see [41]).

After the photon emission the electron spin state is determined by the spin-resolved emission probabilities, integrating the photon polarization in Eq. (1) and instantaneously collapsing into one of its basis states defined with respect to the instantaneous SQA, which is chosen according to the particular observable of interest. To determine the polarization of the electron along the magnetic field in its rest frame, the SQA is chosen along the magnetic field $\boldsymbol{n}_{B}=\boldsymbol{\beta} \times \hat{\mathbf{a}}$ with the scaled electron velocity $\boldsymbol{\beta}$ and the unit vector $\hat{\mathbf{a}}$ [54,57]. In the case when the electron beam is initially polarized with the initial spin vector $\mathbf{S}_{i}$, the observable of interest is the spin expectation value along the initial polarization and the SQA is chosen along that direction [41]. Between photon emissions, the spin precession is governed by the TBMT equation

$$
\begin{aligned}
\left(\frac{d \mathbf{S}}{d \eta}\right)_{T}= & \frac{e \gamma_{e}}{k \cdot p_{i}} \mathbf{S} \times\left[-\left(\frac{g}{2}-1\right) \frac{\gamma_{e}}{\gamma_{e}+1}(\boldsymbol{\beta} \cdot \mathbf{B}) \cdot \boldsymbol{\beta}\right. \\
& \left.+\left(\frac{g}{2}-1+\frac{1}{\gamma_{e}}\right) \mathbf{B}-\left(\frac{g}{2}-\frac{\gamma_{e}}{\gamma_{e}+1}\right) \boldsymbol{\beta} \times \mathbf{E}\right],
\end{aligned}
$$

where $\mathbf{E}$ and $\mathbf{B}$ are the laser electric and magnetic fields, respectively, and the electron gyromagnetic factor $g(\chi)=2+$ $2 \mu(\chi)$, with $\mu(\chi)=\frac{\alpha}{\pi \chi} \int_{0}^{\infty} \frac{y}{(1+y)^{3}} \mathbf{L}_{1 / 3}\left(\frac{2 y}{3 \chi}\right) d y$ and $\mathbf{L}_{1 / 3}(z)=$ $\int_{0}^{\infty} \sin \left[\frac{3 z}{2}\left(x+\frac{x^{3}}{3}\right)\right] d x$ [54]. The simulation results of the electron spin dynamics with our method concur with those of the CAIN code [81].

The electron dynamics in the external laser field between photon emissions is governed by the Lorentz equation 
$d \mathbf{p} / d t=-e(\mathbf{E}+\boldsymbol{\beta} \times \mathbf{B})$. The photon emission induces the electron momentum change $\mathbf{p}_{f} \approx\left(1-\omega_{\gamma} /\left|\mathbf{p}_{i}\right|\right) \mathbf{p}_{i}$, where $\mathbf{p}_{i, f}$ is the electron momentum before and after the emission, respectively.

Note that we have carried out verification of the angular momentum conservation in the considered process. We show that the sum of the orbital and spin angular momenta of the initial electrons and absorbed laser photons equals the total angular (spin) momenta of final electrons and $\gamma$ photons. Although in our simulation the laser field is an external classical field, we estimate the number of absorbed photons in each $\gamma$ photon emission process and confirm the angular momentum conservation by applying the method of Ref. [82].

\section{B. The SF method}

In our SF method, we revise the TBMT equation, including a term responsible for the radiation reaction. For the revision we generalize for arbitrary $\chi$ the method of Refs. [48,70], where the radiation reaction for the spin evolution was calculated at $\chi \ll 1$. Thus, the equation which is used for the spin evolution including the radiation reaction reads

$$
\frac{d \mathbf{S}}{d \eta}=\left(\frac{d \mathbf{S}}{d \eta}\right)_{T}-P\left[\psi_{1}(\chi) \mathbf{S}+\psi_{2}(\chi)(\mathbf{S} \cdot \boldsymbol{\beta}) \boldsymbol{\beta}+\psi_{3}(\chi) \boldsymbol{n}_{B}\right],
$$

where $P=\alpha m^{2} / \sqrt{3} \pi\left(k \cdot p_{i}\right), \quad \psi_{1}(\chi)=\int_{0}^{\infty} u^{\prime \prime} d u K_{2 / 3}\left(u^{\prime}\right)$, $\psi_{2}(\chi)=\int_{0}^{\infty} u^{\prime \prime} d u \int_{u^{\prime}}^{\infty} d x K_{1 / 3}(x)-\psi_{1}(\chi), \psi_{3}(\chi)=\int_{0}^{\infty} u^{\prime \prime} d u$ $K_{1 / 3}\left(u^{\prime}\right)$, and $u^{\prime \prime}=u^{2} /(1+u)^{3}$. As $\chi \ll 1$ the electron dynamics is described by the Landau-Lifshitz equation [83], adding a classical radiative force $\boldsymbol{F}_{c}$ in the Lorentz equation, with

$$
\begin{aligned}
\boldsymbol{F}_{c}= & \frac{2 e^{3}}{3 m}\left\{\gamma_{e}\left[\left(\frac{\partial}{\partial t}+\frac{\mathbf{p}}{\gamma_{e} m} \cdot \nabla\right) \mathbf{E}+\frac{\mathbf{p}}{\gamma_{e} m} \times\left(\frac{\partial}{\partial t}+\frac{\mathbf{p}}{\gamma_{e} m} \cdot \nabla\right) \mathbf{B}\right]\right. \\
& +\frac{e}{m}\left[\mathbf{E} \times \mathbf{B}+\frac{1}{\gamma_{e} m} \mathbf{B} \times(\mathbf{B} \times \mathbf{p})+\frac{1}{\gamma_{e} m} \mathbf{E}(\mathbf{p} \cdot \mathbf{E})\right] \\
& \left.-\frac{e \gamma_{e}}{m^{2}} \mathbf{p}\left[\left(\mathbf{E}+\frac{\mathbf{p}}{\gamma_{e} m} \times \mathbf{B}\right)^{2}-\frac{1}{\gamma_{e}^{2} m^{2}}(\mathbf{E} \cdot \mathbf{p})^{2}\right]\right\}
\end{aligned}
$$

In the employed interaction regime the electrons are ultrarelativistic; consequently, in Eq. (8) we can keep only the leading term with respect to $\gamma_{e}$, namely, the last term (proportional to $\gamma_{e}^{2}$ ), which dominates over the preceding terms [84]. As $\chi \gtrsim 1$, the classical radiation-reaction force in Eq. (8) overestimates the total radiation power, because quantum corrections decrease the average energy emitted by the electron compared to the classical one. Nevertheless, the classical radiationreaction force can be modified in such a way that it will yield radiation losses corresponding to the quantum regime [23]. For this purpose the classical radiation-reaction force is scaled by a function $h(\chi)$ and suppressed appropriately at large $\chi$,

$$
\boldsymbol{F}_{q}=h(\chi) \boldsymbol{F}_{c}
$$

where $h(\chi)=I_{\mathrm{QED}} / I_{C}, \quad I_{\mathrm{QED}}=m \int\left(k \cdot k^{\prime}\right) \frac{d^{2} \bar{W}_{f i}}{d \eta d u} d u, \quad I_{C}=$ $\frac{2 e^{4} E^{2}}{3 m^{2}}, \bar{W}_{f i}$ is the initial spin-resolved radiation probability in Eq. (1) summing over the photon polarization and the electron final spin after radiation, and $E^{\prime}$ is the electric field in the electron rest frame. In the modified Landau-Lifshitz equation, the recoil effects are included by rescaling the radiation-reaction force by the factor $I_{\mathrm{QED}} / I_{C}$, the ratio of the radiation intensities within QED and classical approaches. For ultrarelativistic electrons $\gamma_{e} \gg 1$ the radiative force $\boldsymbol{F}_{c}$ in Eq. (8) is much larger than the spin force (proportional to $\left.\gamma_{e}\right)[84,85]$; thus, the latter is neglected in our simulations. The photon polarization is calculated in this model via the average Stokes parameters $\xi_{1}^{\text {mix }}=F_{1} / F_{0}, \xi_{2}^{\text {mix }}=F_{2} / F_{0}$, and $\xi_{3}^{\operatorname{mix}}=F_{3} / F_{0}[41]$.

We underline that the stochasticity effects due to the quantum radiation reaction originate from discrete photon emissions, taking place as a stochastic process governed by quantum probabilities. As a result of the discrete stochastic photon emission, the momentum of the electron, as well as the spin of the electron, changes discretely and stochastically, creating stochasticity in the momentum and spin dynamics. Consequently, there is no separate stochastic effect on the electron momentum or on the electron spin. Both effects originate from the photon emission and both effects coexist. As $\chi \ll 1$ the stochasticity effects are negligible [23], and the SF and MC models will give similar results.

\section{RESULTS AND DISCUSSION}

\section{A. Case of an initially polarized electron beam}

The angle- and energy-resolved distributions of the polarization and density of the electron beam are illustrated in Fig. 2, including and excluding radiative stochasticity, calculated by the MC and SF methods, respectively. Laser and electron-beam parameters are employed as follows. A realistic tightly focused Gaussian linearly polarized laser pulse [86] propagates along the $+z$ direction (polar angle $\theta_{l}=$ $0^{\circ}$ ), with peak intensity $I_{0} \approx 3.45 \times 10^{21} \mathrm{~W} / \mathrm{cm}^{2}\left(a_{0}=50\right)$, wavelength $\lambda_{0}=1 \mu \mathrm{m}$, pulse duration $\tau=10 T_{0}$ with period $T_{0}$, and focal radius $w_{0}=5 \mu \mathrm{m}$. The counterpropagating LSP electron beam has a cylindrical form, with average spin (polarization) components $\left(\bar{S}_{x}, \bar{S}_{y}, \bar{S}_{z}\right)=(0,0,1)$, polar angle $\theta_{e}=180^{\circ}$, azimuthal angle $\phi_{e}=0^{\circ}$, radius $w_{e}=\lambda_{0}$, length $L_{e}=5 \lambda_{0}$, electron number $N_{e}=5 \times 10^{6}$ (density $n_{e} \approx$ $3.18 \times 10^{17} \mathrm{~cm}^{-3}$ with a transversely Gaussian and longitudinally uniform distribution), initial kinetic energy $\varepsilon_{0}=4$ $\mathrm{GeV}$ (the maximum value of the quantum parameter during the interaction is $\chi_{\max } \approx 1.89$ ), angular divergence $\Delta \theta=0.3$ mrad, energy spread $\Delta \varepsilon_{0} / \varepsilon_{0}=0.06$, and emittance $\epsilon_{e} \approx 3 \times$ $10^{-4} \mathrm{~mm}$ mrad. Such electron beams are achievable via laser wake-field acceleration $[87,88]$ with further radiative polarization $[54,58,59]$ or, alternatively, via directly wake-field acceleration of LSP electrons [64,65].

Radiative stochasticity induces very broad angular and energy distributions in the MC model in comparison with the SF case [cf. Figs. 2(a) and 2(b) with Figs. 2(e) and 2(f)]. The spreads are particularly large in the laser polarization direction. The spin and density distributions of the electrons are demonstrated more visibly for the MC model in Fig. 2(c), by summing over $\theta_{x}$ in Figs. 2(a) and 2(b), respectively. The electron energies after the interaction are distributed in the 

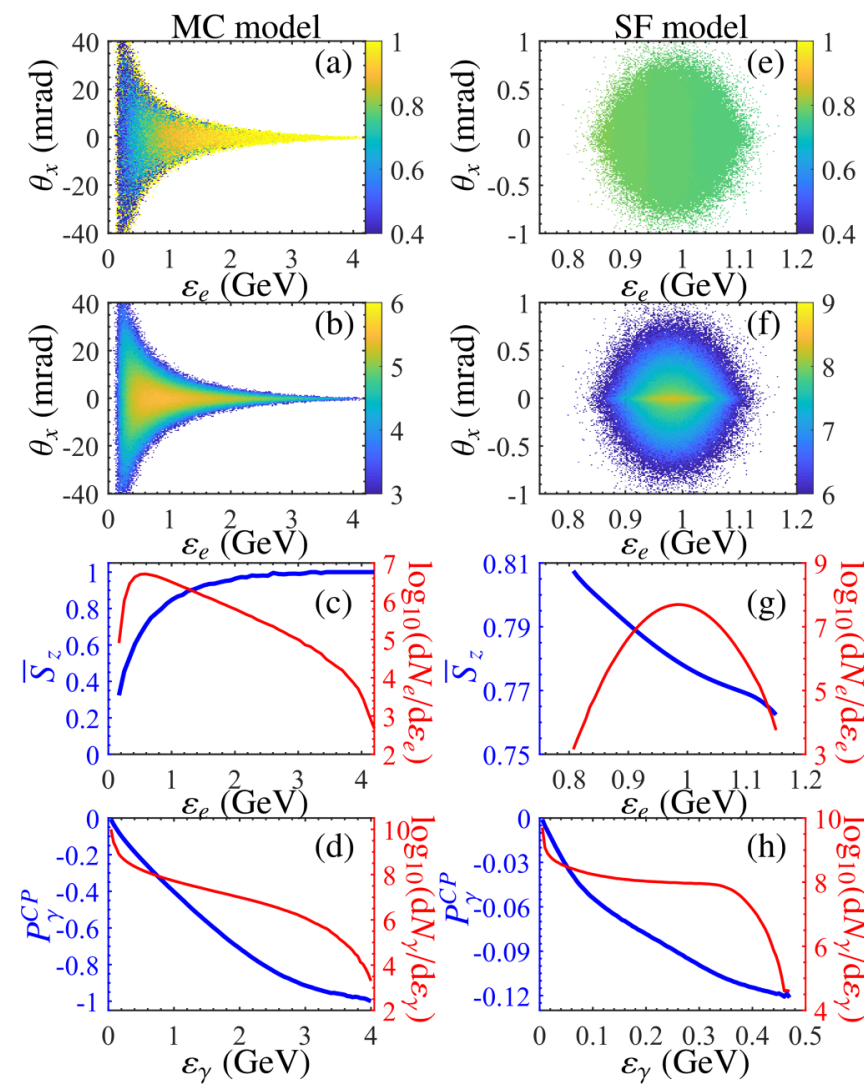

FIG. 2. (a) and (e) Longitudinal average spin (polarization) $\bar{S}_{z}$ vs the deflection angle $\theta_{x}=\arctan \left(p_{x} / p_{z}\right)$ and the electron energy $\varepsilon_{e}$. (b) and (f) Angle-resolved electron density $\log _{10}\left(d^{2} N_{e} / d \theta_{x} d \varepsilon_{e}\right)$ $\left(\mathrm{mrad}^{-1} \mathrm{GeV}^{-1}\right)$. (c) and (g) Plot of $\bar{S}_{z}$ [blue, calculated by summing over $\theta_{x}$ in (a) and (e), respectively] and $\log _{10}\left(d N_{e} / d \varepsilon_{e}\right)$ [red, calculated by summing over $\theta_{x}$ in (b) and (f), respectively] vs $\varepsilon_{e}$. (d) and (h) Degree of circular polarization of emitted photons $P_{\gamma}^{C P}=\xi_{2}$ [41,77] (blue) and energy density $\log _{10}\left(d N_{\gamma} / d \varepsilon_{\gamma}\right)$ (red) vs the photon energy $\varepsilon_{\gamma}$. The left and right columns indicate the cases including and excluding radiative stochasticity, calculated by the MC and SF methods, respectively. The laser and electron-beam parameters are given in the text.

MC simulation in a rather large range from 0.2 to $4.2 \mathrm{GeV}$ because, due to the straggling effects, some electrons do not radiate much. The average spin polarization $\bar{S}_{z}$ monotonically increases with the energy from approximately $34 \%$ up to $100 \%$. This is because more photon emissions lead to more energy losses and more radiation reactions lead to more spin flips and further larger depolarization.

In contrast, in the SF model the final electron energies have a relatively small spread, from approximately $0.81 \mathrm{GeV}$ to $1.15 \mathrm{GeV}$. The $\bar{S}_{z}$ behavior is qualitatively opposite to the MC model; it monotonically decreases with the energy increase, but the variation is not large, from approximately $80.7 \%$ to $76.4 \%$, as shown in Fig. $2(\mathrm{~g})$. We analyze the reason for the polarization behavior with the help of Fig. 3. First of all, let us note that the electrons in the beam experience similar instantaneous laser fields because the applied waist size of the beam is not small $w_{0}=5 w_{e}$ [see the fields experienced by three sample electrons in Fig. 3(b)]. Then the electron dynamics is gradually altered by continuous similar photon emissions. The
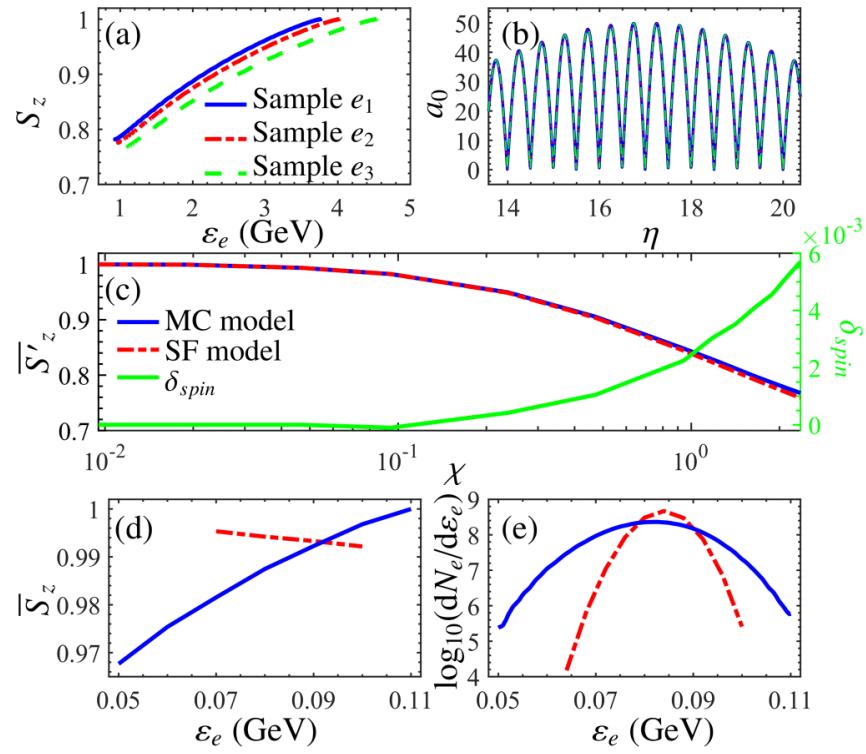

FIG. 3. (a) and (b) Instantaneous $S_{z}$ vs $\varepsilon_{e}$ and experienced $a_{0}$ vs $\eta$ for three sample electrons, respectively, simulated by the SF method. The sample electrons are chosen with random spatial coordinates and different energies. (c) Variation of the average polarization of all electrons $\bar{S}_{z}^{\prime}$ and the relative deviation $\delta_{\text {spin }}$ with respect to $\chi$. Also shown are (d) $\bar{S}_{z}$ and (e) $\log _{10}\left(d N_{e} / d \varepsilon_{e}\right)$ vs $\varepsilon_{e}$ for the case of $\chi \approx 0.047$. In (c)-(e) the blue solid and red dash-dotted curves are simulated by the MC and SF methods, respectively. Other laser and electron-beam parameters are the same as those in Fig. 2.

relation of the polarization to the energy during the interaction is shown in Fig. 3(a). For the electron with a higher initial energy [see the sample electron $e_{3}$ in Fig. 3(a)], the radiation is stronger due to the larger parameter $\chi \sim a_{0} \gamma_{e}$ and consequently the depolarization is larger, but its final energy is still higher because the radiative energy loss is smaller than the initial energy spread.

The average polarization of all electrons $\bar{S}_{z}^{\prime}$ in the MC and SF models are comparable, $\bar{S}_{z}^{\text {MC }} \approx 78.64 \%$ and $\bar{S}_{z}^{\prime S F} \approx$ $77.92 \%$, respectively, derived from data of Figs. 2(c) and $2(\mathrm{~g})$. The relative deviation is $\delta_{\text {spin }}=\left(\bar{S}_{z}^{\prime \mathrm{MC}}-\bar{S}_{z}^{\prime \mathrm{SF}}\right) /\left(\bar{S}_{z}^{\prime \mathrm{MC}}+\right.$ $\left.\bar{S}_{z}^{\prime S F}\right) \approx 0.46 \%$. The variation of $\bar{S}_{z}^{\prime}$ with respect to the quantum parameter $\chi$ is shown in Fig. 3(c), which confirms that the SF method can provide the average depolarization (polarization) degree quite accurately, with a relative error of $\delta_{\text {spin }}<1 \%$ at $\chi \lesssim 2$. With increasing $\chi$, the stochasticity effects become larger and $\delta_{\text {spin }}$ increases. However, at rather low $\chi \approx 0.047$, when the stochasticity is very weak, the average polarization can be deduced from the SF model, but the detailed energy-resolved polarization and density still show differences with respect to the stochastic MC model, as shown in Figs. 3(d) and 3(e). Thus, the increasing behavior of the electron polarization with the energy increase in the electron beam after the interaction [cf. Figs. 2(c) and 2(g)] is a distinct signature of the stochasticity in the radiative depolarization process.

Note that in experiments an appropriate polarimetry is required to measure the average polarization of electrons. The polarization of relativistic electrons can be detected via asymmetries in the momentum distribution of the scattered 

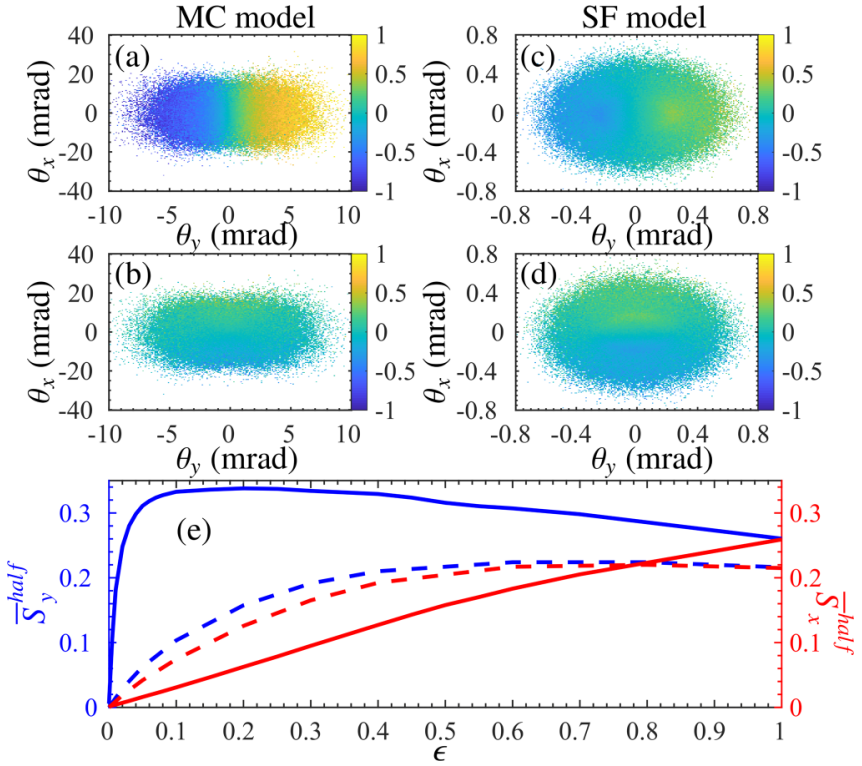

FIG. 4. Transverse polarization components $\bar{S}_{y}$ and $\bar{S}_{x}$ vs the deflection angles $\theta_{x}$ and $\theta_{y}=\arctan \left(p_{y} / p_{z}\right)$, respectively, simulated by (a) and (b) the MC method and (c) and (d) the SF method with the laser ellipticity $\epsilon=\left|E_{y}\right| /\left|E_{x}\right|=0.2$. (e) Average transverse polarization $\bar{S}_{y}^{\text {half }}$ [calculated by summing $\bar{S}_{y}$ over $\theta_{y}>0$ and $\theta_{x}$ in (a) and (c) for the MC (blue solid line) and SF (blue dashed line) methods, respectively] and $\bar{S}_{x}^{\text {half }}$ [calculated by summing $\bar{S}_{x}$ over $\theta_{x}>0$ and $\theta_{y}$ in (b) and (d) for the MC (red solid line) and SF (red dashed line) methods, respectively] vs $\epsilon$. Other laser and electron-beam parameters are the same as those in Fig. 2.

electrons or emitted photons. Polarimetry commonly employs the following physical principles: Mott scattering [89], Møller scattering [90], synchrotron radiation [91], and linear [92] and nonlinear Compton scattering [56]. The Mott and Møller polarimetries are inapplicable at high energies $(\gtrsim 10 \mathrm{MeV})$ [93] and at high currents $(\gtrsim 100 \mu \mathrm{A})$ [94], respectively. The polarimetry based on synchrotron radiation demands a largescale synchrotron facility. The linear Compton polarimetry is suitable at the considered high energies; however, it requires a large number of laser shots to reach a small statistical uncertainty of approximately $1 \%$ due to low electron-photon collision luminosity. With currently achievable ultraintense laser facilities, more promising is the nonlinear Compton polarimetry, which can provide competitive resolution in a single-shot setup [56].

We have investigated also the role of stochasticity effects for emitted high-energy highly circularly polarized $\gamma$ rays [see Figs. 2(d) and 2(h)]. While the circular polarization degree of $\gamma$ photons varies with energy in a rather large range from approximately 0 to -1 in the MC model, the SF model shows a much smaller range from approximately 0 to -0.1 . However, the average polarization degrees are similar and low, about -0.077 and -0.081 for the MC and SF models, respectively. This is because in the MC model the polarization is high for high-energy photons with very low numbers [see Figs. 2(d)-(h)]. The energy range of $\gamma$ photons is much larger in the MC model, similar to the electron energy distribution,
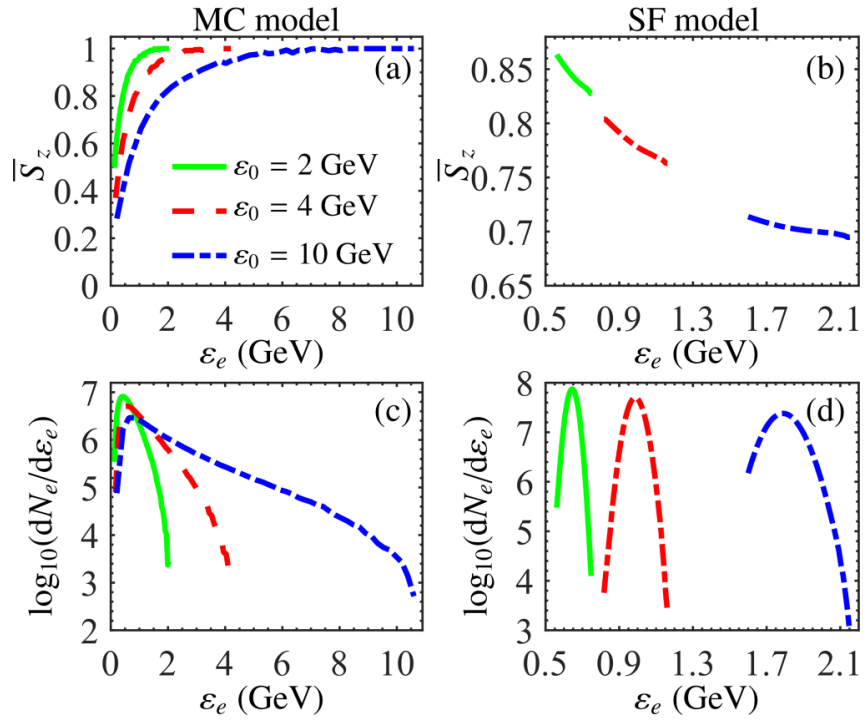

FIG. 5. Plot of $\bar{S}_{z}$ vs $\varepsilon_{e}$, simulated by the (a) MC and (b) SF methods, and plot of $\log _{10}\left(d N_{e} / d \varepsilon_{e}\right)$ vs $\varepsilon_{e}$, simulated by the (c) MC and (d) SF methods. The green solid, red dashed, and blue dashdotted curves indicate the cases of the initial kinetic energy of the electron beam $\varepsilon_{0}=2,4$, and $10 \mathrm{GeV}$, respectively. Other laser and electron-beam parameters are the same as those in Fig. 2.

which yields generation of high-energy high-brilliance highly circularly polarized $\gamma$ rays, as discussed in [41].

\section{B. Case of an initially unpolarized electron beam}

Now we turn to the discussion of the case of an initially unpolarized electron beam and look for the stochasticity signatures in the spin dynamics. It is known [54] that an initially unpolarized electron beam can be split into two oppositely polarized parts during interaction with a counterpropagating elliptically polarized laser pulse (the minor axis along the $y$ direction). We have analyzed this polarization-dependent splitting effect with the MC and SF models for the full range of ellipticity (see Fig. 4). Exemplary distributions of the transverse polarization components with respect to the electron deflection angle after the interaction in the case of $\epsilon=0.2$ are shown in Figs. 4(a)-4(d), calculated within the MC and SF models, respectively. In both models the electron beam splits into two parts along the propagation direction, which are oppositely polarized. At small ellipticity, the electron spin polarization along the minor axis of the ellipticity is the largest, with small angular separation along that axis. The separated half of the electron beam (e.g., $\theta_{y}>0$ ) has an average polarization (e.g., $\bar{S}_{y}^{\text {half }}$ ), which depends on the separation angle: The larger the separation angle, the larger the average polarization $\bar{S}_{y}^{\text {half }}$. In the MC model the separation angle is significantly larger than that in the SF case due to stochasticity (as in this case photons of larger energies are emitted) and consequently $\bar{S}_{y}^{\text {half }}$ is larger [see Fig. 4(e)]. The deviation of $\bar{S}_{y}^{\text {half }}$ between the MC and SF models is the largest at small ellipticity near 0.05 for the given parameters. In the MC model of Fig. 4(a), $\left|\bar{S}_{y}^{\text {half }}\right| \approx 33.8 \%$; by comparison, in the SF model 

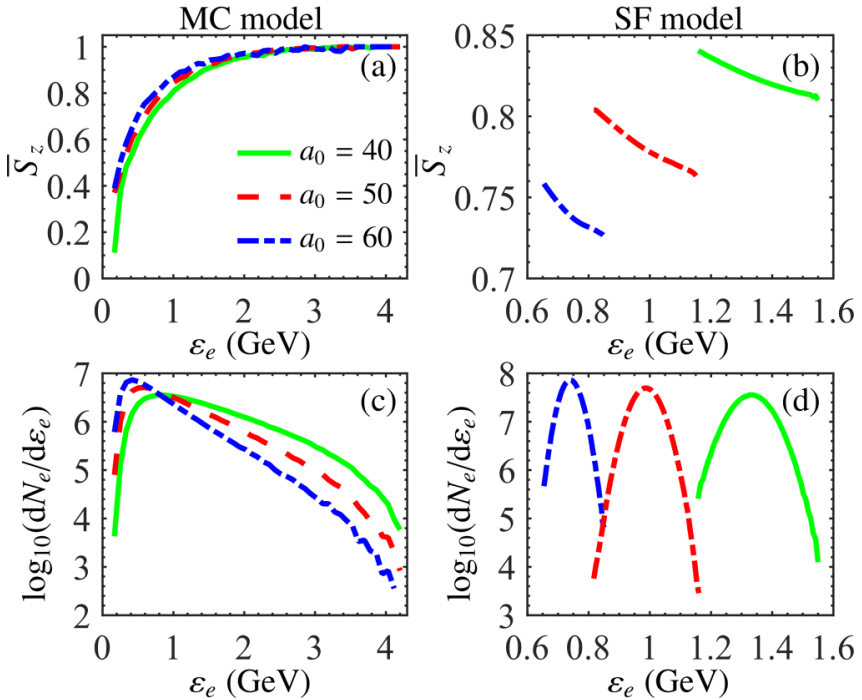

FIG. 6. Plot of $\bar{S}_{z}$ vs $\varepsilon_{e}$, simulated by the (a) MC and (b) SF methods, and plot of $\log _{10}\left(d N_{e} / d \varepsilon_{e}\right)$ vs $\varepsilon_{e}$, simulated by the (c) MC and (d) SF methods. The green solid, red dashed, and blue dash-dotted curves indicate the cases of the invariant field parameter $a_{0}=40$, 50 , and 60 , respectively. Other laser and electron-beam parameters are the same as those in Fig. 2.

of Fig. 4(c), $\left|\bar{S}_{y}^{\text {half }}\right| \approx 15.8 \%$ is much lower. While in the SF model $\bar{S}_{x}^{\text {half }}$ and $\bar{S}_{y}^{\text {half }}$ increase monotonically with an increase of the ellipticity, in the MC model $\bar{S}_{y}^{\text {half }}$ demonstrates a characteristic nonmonotonic behavior with a peak at small ellipticity. The latter can serve as a signature of the stochasticity effects in radiative polarization of initially unpolarized electron beams.
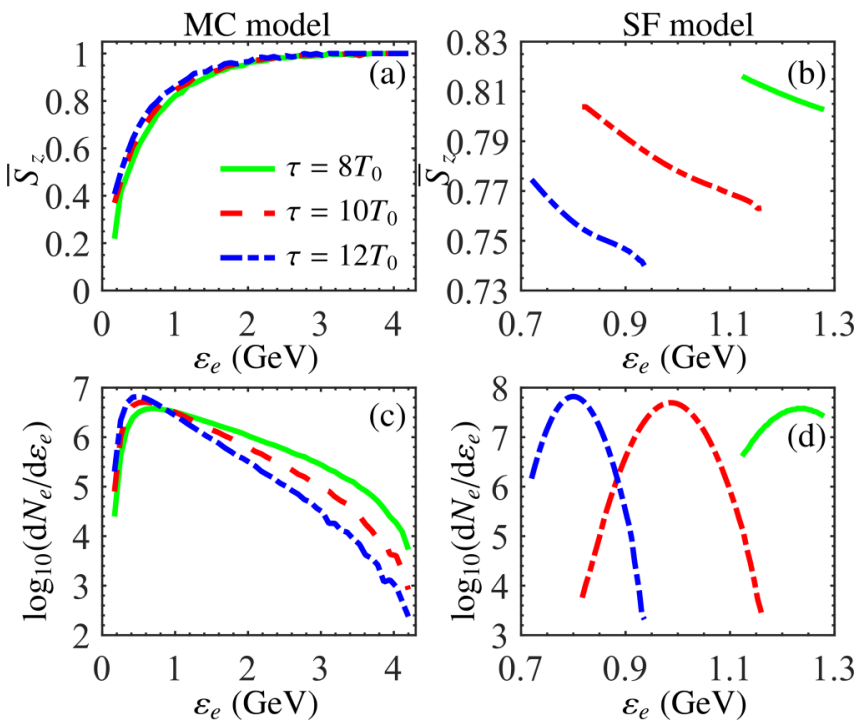

FIG. 7. Plot of $\bar{S}_{z}$ vs $\varepsilon_{e}$, simulated by the (a) MC and (b) SF methods, and plot of $\log _{10}\left(d N_{e} / d \varepsilon_{e}\right)$ vs $\varepsilon_{e}$, simulated by the (c) MC and (d) SF methods. The green solid, red dashed, and blue dash-dotted curves indicate the cases of the laser pulse duration $\tau=8 T_{0}, 10 T_{0}$, and $12 T_{0}$, respectively. Other laser and electron-beam parameters are the same as those in Fig. 2.
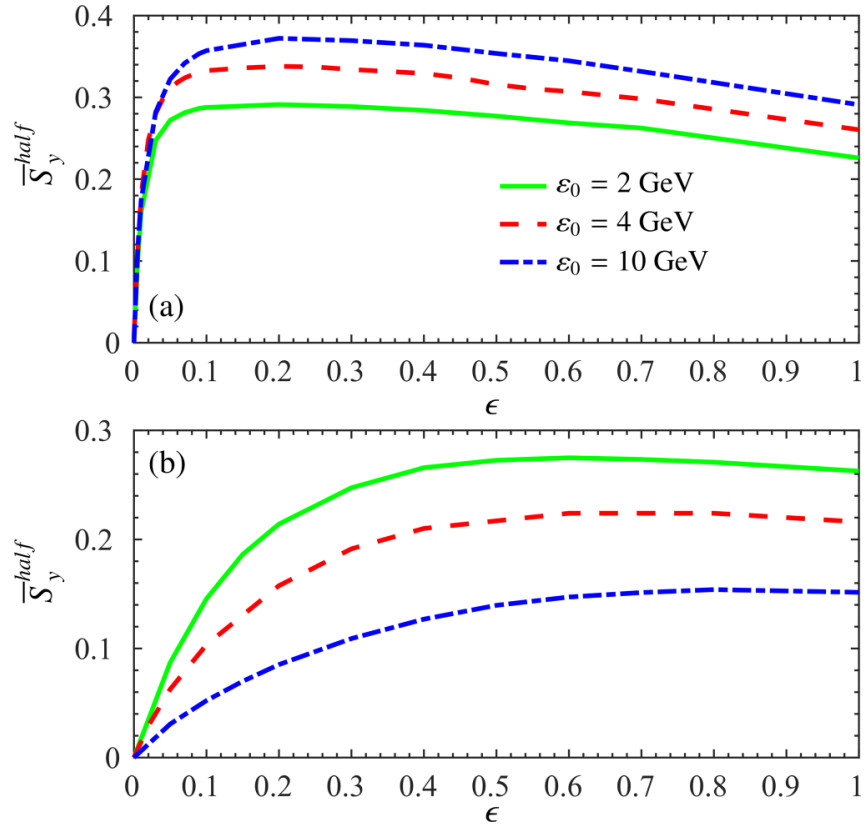

FIG. 8. Plot of $\bar{S}_{y}^{\text {half }}$ vs $\epsilon$, simulated by the (a) MC and (b) SF methods. The green solid, red dashed, and blue dash-dotted curves indicate the cases of $\varepsilon_{0}=2,4$, and $10 \mathrm{GeV}$, respectively. Other laser and electron-beam parameters are the same as those in Fig. 4.

\section{Impact of the laser and electron-beam parameters on the considered signatures}

For experimental feasibility, we investigate the impact of the laser and electron-beam parameters, e.g., variations of the initial kinetic energy of the electron beam $\varepsilon_{0}$, the invariant
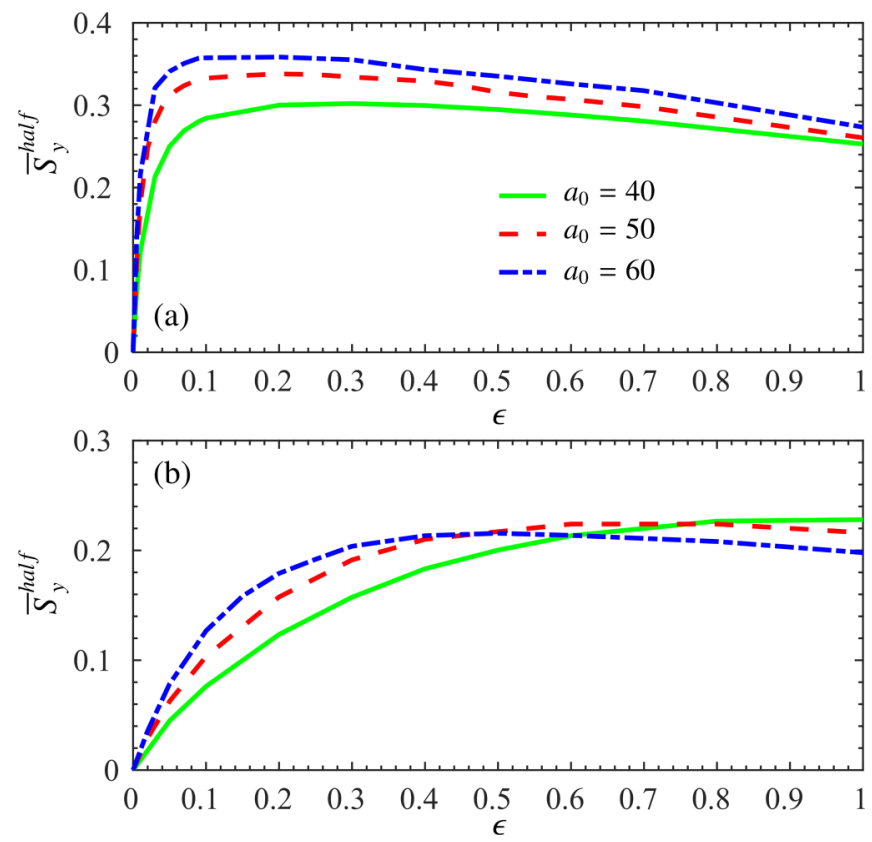

FIG. 9. Plot of $\bar{S}_{y}^{\text {half }}$ vs $\epsilon$, simulated by the (a) MC and (b) SF methods. The green solid, red dashed, and blue dash-dotted curves indicate the cases of $a_{0}=40,50$, and 60, respectively. Other laser and electron-beam parameters are the same as those in Fig. 4. 

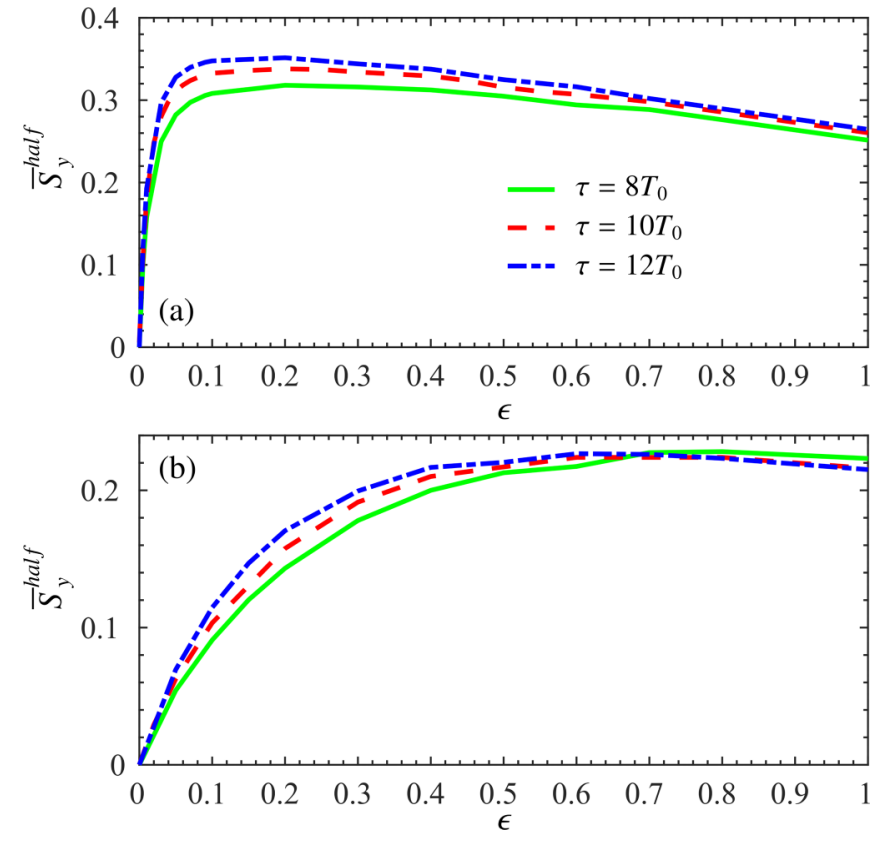

FIG. 10. Plot of $\bar{S}_{y}^{\text {half }}$ vs $\epsilon$, simulated by the (a) MC and (b) SF methods. The green solid, red dashed, and blue dash-dotted curves indicate the cases of $\tau=8 T_{0}, 10 T_{0}$, and $12 T_{0}$, respectively. Other laser and electron-beam parameters are the same as those in Fig. 4.

field parameter $a_{0}$, and the laser pulse duration $\tau$, on the considered signatures of radiative stochasticity. Corresponding to Fig. 2, as $\varepsilon_{0}$ increases from $2 \mathrm{GeV}$ to $10 \mathrm{GeV}, a_{0}$ from 40 to 60 , and $\tau$ from $8 T_{0}$ to $12 T_{0}$, the considered signatures of radiative stochasticity remain uniform, as shown in Figs. 5-7. In addition, with the variations of $\varepsilon_{0}, a_{0}$, and $\tau$, the considered signatures of radiative stochasticity corresponding to Fig. 4 remain qualitatively the same, as shown in Fig. 4. For instance, with increases of $\varepsilon_{0}$ and $a_{0}$, the stochasticity effects are enhanced since $\chi \propto a_{0} \varepsilon_{0}$ increases in the MC model and consequently the peak of $\bar{S}_{y}^{\text {half }}$ goes up [see Figs. 8(a) and 9(a)]. However, in the SF model, as $\varepsilon_{0}\left(a_{0}\right)$ increases, the splitting angle $\theta \sim a_{0} / \varepsilon_{0}$ decreases (increases); thus $\bar{S}_{y}^{\text {half }}$ goes down (up) at small $\epsilon$, and this effect is weakened and even eliminated at large $\epsilon$ due to the laser field rotation [see Figs. 8(b) and 9(b)]. As $\tau$ increases, $\bar{S}_{y}^{\text {half }}$ increases at small $\epsilon$ due to more photon emissions $\sim \tau$, as demonstrated in Fig. 10. The variation of the energy spread of the electron beam does not change the considered qualitative signatures either.
To summarize, we developed a semiclassical stochasticityfree method of radiative polarization applicable in the quantum regime for arbitrary $\chi$. Then we revealed qualitative signatures of the stochastic effects on electron spin, which are much different from those well studied for electron momenta, are feasible with the currently available laser facilities, and provide a measurable objective for future strong laser experiments.

\section{CONCLUSION}

Photon emission by an electron in the quantum regime is a discrete stochastic process, which leaves its signatures in the momentum as well as in the spin dynamics of the electron. We have shown that the stochastic photon emission will induce qualitative changes in the angle- and energy-resolved average spin distributions with respect to the stochasticity-free case. We have analyzed the impact of stochastic photon emission in a strong laser field on the initially LSP electron radiative depolarization as well as on the emitted $\gamma$-ray polarization. To display the stochasticity on the electron spin, we have developed a semiclassical SF method of radiative polarization applicable in the quantum regime for arbitrary $\chi$. The qualitative signatures of the stochasticity have been demonstrated in the energy-resolved electron polarization after the interaction and in the energy-resolved polarization of the emitted $\gamma$ photons. In the case of an initially unpolarized electron beam, the stochasticity effect was demonstrated in the dependence of the electron polarization on the laser ellipticity. These qualitative signatures are observable with the currently available laser facilities and are robust with respect to the laser and electron-beam parameters. They extend our understanding of the stochastic effects at photon emissions, which have previously been known only as yielding a broadening of the energy spread of an electron beam.

\section{ACKNOWLEDGMENTS}

R.-T.G., Y.W., and J.-X.L. thank Prof. C. Keitel for hospitality. This work was supported by the National Natural Science Foundation of China (Grants No. 11874295, No. 11875219, and No. 11905169) and the National Key R\&D Program of China (Grant No. 2018YFA0404801). Y.-Y.C. was supported by the Program for Professor of Special Appointment (Eastern Scholar) at Shanghai Institutions of Higher Learning and Shanghai Rising-Star Program.
[1] C. N. Danson, C. Haefner, J. Bromage, T. Butcher, J.-C. F. Chanteloup, E. A. Chowdhury, A. Galvanauskas, L. A. Gizzi, J. Hein, D. I. Hillier et al., Petawatt and exawatt class lasers worldwide, High Power Laser Sci. Eng. 7, e54 (2019).

[2] J. W. Yoon, C. Jeon, J. Shin, S. K. Lee, H. W. Lee, I. W. Choi, H. T. Kim, J. H. Sung, and C. H. Nam, Achieving the laser intensity of $5.5 \times 10^{22} \mathrm{~W} / \mathrm{cm}^{2}$ with a wavefront-corrected multi-PW laser, Opt. Express 27, 20412 (2019).
[3] S. Gales, K. A. Tanaka, D. L. Balabanski, F. Negoita, D. Stutman, O. Tesileanu, C. A. Ur, D. Ursescu, I. Andrei, S. Ataman et al., The extreme light infrastructure-nuclear physics (ELI-NP) facility: New horizons in physics with $10 \mathrm{PW}$ ultraintense lasers and $20 \mathrm{MeV}$ brilliant gamma beams, Rep. Prog. Phys. 81, 094301 (2018).

[4] ELI Beamlines, http://www.eli-beams.eu/en/facility/lasers/.

[5] Vulcan laser facility, http://www.clf.stfc.ac.uk/Pages/TheVulcan-10-Petawatt-Project.aspx. 
[6] Exawatt Center for Extreme Light Studies, http://www.xcels. iapras.ru/.

[7] Center for Relativistic Laser Science, https://www.ibs.re.kr/ eng/sub02_03_05.do.

[8] V. I. Ritus, Quantum effects of the interaction of elementary particles with an intense electromagnetic field, J. Sov. Laser Res. 6, 497 (1985).

[9] C. Bula, K. T. McDonald, E. J. Prebys, C. Bamber, S. Boege, T. Kotseroglou, A. C. Melissinos, D. D. Meyerhofer, W. Ragg, D. L. Burke, R. C. Field, G. Horton-Smith, A. C. Odian, J. E. Spencer, D. Walz, S. C. Berridge, W. M. Bugg, K. Shmakov, and A. W. Weidemann, Observation of Nonlinear Effects in Compton Scattering, Phys. Rev. Lett. 76, 3116 (1996).

[10] D. L. Burke, R. C. Field, G. Horton-Smith, J. E. Spencer, D. Walz, S. C. Berridge, W. M. Bugg, K. Shmakov, A. W. Weidemann, C. Bula, K. T. McDonald, E. J. Prebys, C. Bamber, S. J. Boege, T. Koffas, T. Kotseroglou, A. C. Melissinos, D. D. Meyerhofer, D. A. Reis, and W. Ragg, Positron Production in Multiphoton Light-by-Light Scattering, Phys. Rev. Lett. 79, 1626 (1997).

[11] G. Sarri, D. J. Corvan, W. Schumaker, J. M. Cole, A. Di Piazza, H. Ahmed, C. Harvey, C. H. Keitel, K. Krushelnick, S. P. D. Mangles, Z. Najmudin, D. Symes, A. G. R. Thomas, M. Yeung, Z. Zhao, and M. Zepf, Ultrahigh Brilliance Multi-MeV $\gamma$-Ray Beams From Nonlinear Relativistic Thomson Scattering, Phys. Rev. Lett. 113, 224801 (2014).

[12] G. Sarri, K. Poder, J. M. Cole, W. Schumaker, A. Di Piazza, B. Reville, T. Dzelzainis, D. Doria, L. A. Gizzi, G. Grittani et al., Generation of neutral and high-density electron-positron pair plasmas in the laboratory, Nat. Commun. 6, 6747 (2015).

[13] W. Yan, C. Fruhling, G. Golovin, D. Haden, J. Luo, P. Zhang, B. Zhao, J. Zhang, C. Liu, M. Chen, S. Chen, S. Banerjee, and D. Umstadter, High-order multiphoton Thomson scattering, Nat. Photonics 11, 514 (2017).

[14] I. I. Goldman, Intensity effects in compton scattering, Sov. Phys. JETP 19, 954 (1964) [Zh. Eksp. Teor. Fiz. 46, 1412 (1964)].

[15] A. I. Nikishov and V. I. Ritus, Quantum processes in the field of a plane electromagnetic wave and in a constant field. I, Sov. Phys. JETP 19, 529 (1964) [Zh. Eksp. Teor. Fiz. 46, 776 (1964)].

[16] L. S. Brown and T. W. B. Kibble, Interaction of intense laser beams with electrons, Phys. Rev. 133, A705 (1964).

[17] H. R. Reiss, Absorption of light by light, J. Math. Phys. 3, 59 (1962).

[18] W. Heitler, The Quantum Theory of Radiation (Clarendon, Oxford, 1954).

[19] I. C. E. Turcu, B. Shen, D. Neely, G. Sarri, K. A. Tanaka, P. McKenna, S. P. D. Mangles, T.-P. Yu, W. Luo, X.-L. Zhu et al., Quantum electrodynamics experiments with colliding petawatt laser pulses, High Power Laser Sci. Eng. 7, e10 (2019).

[20] M. Abraham, Theorie der Elektrizität (Teubner, Leipzig, 1905).

[21] H. A. Lorentz, The Theory of Electrons (Teubner, Leipzig, 1909).

[22] P. A. M. Dirac, Classical theory of radiating electrons, Proc. R. Soc. London Ser. A 167, 148 (1938).

[23] A. Di Piazza, C. Müller, K. Z. Hatsagortsyan, and C. H. Keitel, Extremely high-intensity laser interactions with fundamental quantum systems, Rev. Mod. Phys. 84, 1177 (2012).
[24] J. M. Cole, K. T. Behm, E. Gerstmayr, T. G. Blackburn, J. C. Wood, C. D. Baird, M. J. Duff, C. Harvey, A. Ilderton, A. S. Joglekar et al., Experimental Evidence of Radiation Reaction in the Collision of a High-Intensity Laser Pulse with a Laser-Wakefield Accelerated Electron Beam, Phys. Rev. X 8, 011020 (2018).

[25] K. Poder, M. Tamburini, G. Sarri, A. Di Piazza, S. Kuschel, C. D. Baird, K. Behm, S. Bohlen, J. M. Cole, D. J. Corvan et al., Experimental Signatures of the Quantum Nature of Radiation Reaction in the Field of an Ultraintense Laser, Phys. Rev. X 8, 031004 (2018).

[26] N. Neitz and A. Di Piazza, Stochasticity Effects in Quantum Radiation Reaction, Phys. Rev. Lett. 111, 054802 (2013).

[27] N. Neitz and A. Di Piazza, Electron-beam dynamics in a strong laser field including quantum radiation reaction, Phys. Rev. A 90, 022102 (2014).

[28] S. R. Yoffe, Y. Kravets, A. Noble, and D. A. Jaroszynski, Longitudinal and transverse cooling of relativistic electron beams in intense laser pulses, New J. Phys. 17, 053025 (2015).

[29] A. V. Bashinov, A. V. Kim, and A. M. Sergeev, Impact of quantum effects on relativistic electron motion in a chaotic regime, Phys. Rev. E 92, 043105 (2015).

[30] C. N. Harvey, A. Gonoskov, A. Ilderton, and M. Marklund, Quantum Quenching of Radiation Losses in Short Laser Pulses, Phys. Rev. Lett 118, 105004 (2017).

[31] A. Di Piazza, K. Z. Hatsagortsyan, and C. H. Keitel, Quantum Radiation Reaction Effects in Multiphoton Compton Scattering, Phys. Rev. Lett. 105, 220403 (2010).

[32] J.-X. Li, Y.-Y. Chen, K. Z. Hatsagortsyan, and C. H. Keitel, Angle-resolved stochastic photon emission in the quantum radiation-dominated regime, Sci. Rep. 7, 11556 (2017).

[33] C. S. Shen and D. White, Energy Straggling and Radiation Reaction for Magnetic Bremsstrahlung, Phys. Rev. Lett. 28, 455 (1972).

[34] T. G. Blackburn, C. P. Ridgers, J. G. Kirk, and A. R. Bell, Quantum Radiation Reaction in Laser-Electron-Beam Collisions, Phys. Rev. Lett. 112, 015001 (2014).

[35] T. N. Wistisen and U. I. Uggerhøj, Vacuum birefringence by Compton backscattering through a strong field, Phys. Rev. D 88, 053009 (2013).

[36] A. Ilderton and M. Marklund, Prospects for studying vacuum polarization using dipole and synchrotron radiation, J. Plasma Phys. 82, 655820201 (2016).

[37] B. King and N. Elkina, Vacuum birefringence in high-energy laser-electron collisions, Phys. Rev. A 94, 062102 (2016).

[38] S. Bragin, S. Meuren, C. H. Keitel, and A. Di Piazza, HighEnergy Vacuum Birefringence and Dichroism in an Ultrastrong Laser Field, Phys. Rev. Lett. 119, 250403 (2017).

[39] Y. Nakamiya and K. Homma, Probing vacuum birefringence under a high-intensity laser field with gamma-ray polarimetry at the GeV scale, Phys. Rev. D 96, 053002 (2017).

[40] V. N. Baier, V. M. Katkov, and V. M. Strakhovenko, Electromagnetic Processes at High Energies in Oriented Single Crystals (World Scientific, Singapore, 1998).

[41] Y.-F. Li, R. Shaisultanov, Y.-Y. Chen, F. Wan, K. Z Hatsagortsyan, C. H. Keitel, and J.-X. Li, Polarized Ultrashort Brilliant Multi-GeV $\gamma$ Rays via Single-Shot Laser-Electron Interaction, Phys. Rev. Lett. 124, 014801 (2020). 
[42] V. N. Baier, V. M. Katkov, and V. S. Fadin, Radiation from Relativistic Electrons (Atomizdat, Moscow, 1973).

[43] B. King, N. Elkina, and H. Ruhl, Photon polarization in electron-seeded pair-creation cascades, Phys. Rev. A 87, 042117 (2013).

[44] F. Wan, Y. Wang, R.-T. Guo, Y.-Y. Chen, R. Shaisultanov, Z.F. Xu, K. Z. Hatsagortsyan, C. H. Keitel, and J.-X. Li, Highenergy $\gamma$-photon polarization in nonlinear Breit-Wheeler pair production and $\gamma$ polarimetry, Phys. Rev. Research 2, 032049 (2020).

[45] A. Sokolov and I. Ternov, On polarization and spin effects in the theory of synchrotron radiation, Sov. Phys. Dokl. 8, 1203 (1964).

[46] Synchrotron Radiation, edited by A. A. Sokolov and I. M. Ternov (Nauka, Moscow, 1968).

[47] V. N. Baier and V. M. Katkov, Radiational polarization of electrons in inhomogeneous magnetic field, Phys. Lett. A 24, 327 (1967).

[48] V. N. Baier, Radiative polarization of electron in storage rings, Sov. Phys. Usp. 14, 695 (1972).

[49] Y. S. Derbenev and A. M. Kondratenko, Diffusion of particle spins in storage rings, Sov. Phys. JETP 35, 230 (1972).

[50] K. Heinemann and D. P. Barber, Spin transport, spin diffusion and Bloch equations in electron storage rings, Nucl. Instrum. Methods Phys. Res. Sect. A 463, 62 (2001).

[51] D. Del Sorbo, D. Seipt, T. G. Blackburn, A. G. R. Thomas, C. D. Murphy, J. G. Kirk, and C. P. Ridgers, Spin polarization of electrons by ultraintense lasers, Phys. Rev. A 96, 043407 (2017).

[52] D. Del Sorbo, D. Seipt, A. G. R. Thomas, and C. P. Ridgers, Electron spin polarization in realistic trajectories around the magnetic node of two counter-propagating, circularly polarized, ultra-intense lasers, Plasma Phys. Control. Fusion 60, 064003 (2018).

[53] D. Seipt, D. Del Sorbo, C. P. Ridgers, and A. G. R. Thomas, Theory of radiative electron polarization in strong laser fields, Phys. Rev. A 98, 023417 (2018).

[54] Y.-F. Li, R. Shaisultanov, K. Z. Hatsagortsyan, F. Wan, C. H. Keitel, and J.-X. Li, Ultrarelativistic Electron-Beam Polarization in Single-Shot Interaction with an Ultraintense Laser Pulse, Phys. Rev. Lett. 122, 154801 (2019).

[55] F. Wan, R. Shaisultanov, Y.-F. Li, K. Z. Hatsagortsyan, C. H. Keitel, and J.-X. Li, Ultrarelativistic polarized positron jets via collision of electron and ultraintense laser beams, Phys. Lett. B 800, 135120 (2020).

[56] Y.-F. Li, R.-T. Guo, R. Shaisultanov, K. Z. Hatsagortsyan, and J.-X. Li, Electron Polarimetry with Nonlinear Compton Scattering, Phys. Rev. Appl. 12, 014047 (2019).

[57] Y.-Y. Chen, P.-L. He, R. Shaisultanov, K. Z. Hatsagortsyan, and C. H. Keitel, Polarized Positron Beams via Intense Two-Color Laser Pulses, Phys. Rev. Lett. 123, 174801 (2019).

[58] H.-H. Song, W.-M. Wang, J.-X. Li, Y.-F. Li, and Y.-T. Li, Spinpolarization effects of an ultrarelativistic electron beam in an ultraintense two-color laser pulse, Phys. Rev. A 100, 033407 (2019).

[59] D. Seipt, D. Del Sorbo, C. P. Ridgers, and A. G. R. Thomas, Ultrafast polarization of an electron beam in an intense bichromatic laser field, Phys. Rev. A 100, 061402(R) (2019).

[60] D. T. Pierce and F. Meier, Photoemission of spin-polarized electrons from GaAs, Phys. Rev. B 13, 5484 (1976).
[61] H. Batelaan, A. S. Green, B. A. Hitt, and T. J. Gay, Optically Pumped Electron Spin Filter, Phys. Rev. Lett. 82, 4216 (1999).

[62] M. M. Dellweg and C. Müller, Spin-Polarizing Interferometric Beam Splitter for Free Electrons, Phys. Rev. Lett. 118, 070403 (2017).

[63] M. L. Swartz, Physics with polarized electron beams, SLAC Rep. No. 4656, 1988 (unpublished).

[64] M. Wen, M. Tamburini, and C. H. Keitel, Polarized LaserWakefield-Accelerated Kiloampere Electron Beams, Phys. Rev. Lett. 122, 214801 (2019).

[65] Y. Wu, L. Ji, X. Geng, Q. Yu, N. Wang, B. Feng, Z. Guo, W. Wang, C. Qin, X. Yan, L. Zhang, J. Thomas, A. Hützen, M. Büscher, P. Rakitzis, A. Pukhov, B. Shen, and R. Li, Polarized electron-beam acceleration driven by vortex laser pulses, New J. Phys. 21, 073052 (2019).

[66] Y. Derbenev and A. M. Kondratenko, Polarization kinematics of particles in storage rings, Sov. Phys. JETP 37, 968 (1973).

[67] S. R. Mane, Electron-spin polarization in high-energy storage rings. I. Derivation of the equilibrium polarization, Phys. Rev. A 36, 105 (1987).

[68] D. Y. Ivanov, G. L. Kotkin, and V. G. Serbo, Complete description of polarization effects in emission of a photon by an electron in the field of a strong laser wave, Eur. Phys. J. C 36, 127 (2004).

[69] D. Y. Ivanov, G. L. Kotkin, and V. G. Serbo, Complete description of polarization effects in $e^{+}-e^{-}$pair production by a photon in the field of a strong laser wave, Eur. Phys. J. C 40, 27 (2005).

[70] V. N. Baier, V. M. Katkov, and V. M. Strakhovenko, Kinetics of radiative polarization, Sov. Phys. JETP 31, 908 (1970).

[71] L. H. Thomas, The motion of the spinning electron, Nature (London) 117, 514 (1926).

[72] L. H. Thomas, The kinematics of an electron with an axis, Philos. Mag. 3, 1 (1927).

[73] V. Bargmann, L. Michel, and V. L. Telegdi, Precession of the Polarization of Particles Moving in a Homogeneous Electromagnetic Field, Phys. Rev. Lett. 2, 435 (1959).

[74] A. Ilderton, Note on the conjectured breakdown of QED perturbation theory in strong fields, Phys. Rev. D 99, 085002 (2019).

[75] A. Di Piazza, M. Tamburini, S. Meuren, and C. H. Keitel, Improved local-constant-field approximation for strong-field QED codes, Phys. Rev. A 99, 022125 (2019).

[76] T. G. Blackburn, D. Seipt, S. S. Bulanov, and M. Marklund, Radiation beaming in the quantum regime, Phys. Rev. A 101, 012505 (2020).

[77] W. H. McMaster, Matrix representation of polarization, Rev. Mod. Phys. 33, 8 (1961).

[78] C. P. Ridgers, J. G. Kirk, R. Duclous, T. G. Blackburn, C. S. Brady, K. Bennett, T. D. Arber, and A. R. Bell, Modelling gamma-ray photon emission and pair production in highintensity laser-matter interactions, J. Comput. Phys. 260, 273 (2014).

[79] N. V. Elkina, A. M. Fedotov, I. Y. Kostyukov, M. V. Legkov, N. B. Narozhny, E. N. Nerush, and H. Ruhl, QED cascades induced by circularly polarized laser fields, Phys. Rev. ST Accel. Beams 14, 054401 (2011).

[80] D. G. Green and C. N. Harvey, SIMLA: Simulating particle dynamics in intense laser and other electromagnetic fields via classical and quantum electrodynamics, Computer. Phys. Commun. 192, 313 (2015). 
[81] K. Yokoya, CAIN 2.42 User Manual, https://ilc.kek.jp/ yokoya/ CAIN/Cain242/.

[82] Y.-Y. Chen, J.-X. Li, K. Z. Hatsagortsyan, and C. H. Keitel, $\gamma$-Ray Beams with Large Orbital Angular Momentum via Nonlinear Compton Scattering with Radiation Reaction, Phys. Rev. Lett. 121, 074801 (2018).

[83] L. D. Landau and E. M. Lifshitz, The Classical Theory of Fields (Elsevier, Oxford, 1975).

[84] M. Tamburini, F. Pegoraro, A. Di Piazza, C. H. Keitel, and A. Macchi, Radiation reaction effects on radiation pressure acceleration, New J. Phys. 12, 123005 (2010).

[85] M. Wen, H. Bauke, and C. H. Keitel, Identifying the SternGerlach force of classical electron dynamics, Sci. Rep. 6, 31624 (2016).

[86] Y. I. Salamin and C. H. Keitel, Electron Acceleration by a Tightly Focused Laser Beam, Phys. Rev. Lett. 88, 095005 (2002).

[87] W. P. Leemans, A. J. Gonsalves, H.-S. Mao, K. Nakamura, C. Benedetti, C. B. Schroeder, C. Tóth, J. Daniels, D. E. Mittelberger, S. S. Bulanov, J.-L. Vay, C. G. R. Geddes, and E. Esarey, Multi-GeV Electron Beams from Capillary-DischargeGuided Subpetawatt Laser Pulses in the Self-Trapping Regime, Phys. Rev. Lett. 113, 245002 (2014).
[88] A. J. Gonsalves, K. Nakamura, J. Daniels, C. Benedetti, C. Pieronek, T. C. H. de Raadt, S. Steinke, J. H. Bin, S. S. Bulanov, J. van Tilborg et al., Petawatt Laser Guiding and Electron Beam Acceleration to $8 \mathrm{GeV}$ in a Laser-Heated Capillary Discharge Waveguide, Phys. Rev. Lett. 122, 084801 (2019).

[89] N. F. Mott, The scattering of fast electrons by atomic nuclei, Proc. R. Soc. (London) 124, 425 (1929).

[90] C. Møller, On the theory of the passage of rapid electrons through matter, Ann. Phys. (Leipzig) 406, 531 (1932).

[91] D. P. Barber et al., The HERA polarimeter and the first observation of electron spin polarization at HERA, Nucl. Instrum. Methods Phys. Res. Sect. A 329, 79 (1993).

[92] A. Narayan, D. Jones, J. C. Cornejo, M. M. Dalton, W. Deconinck, D. Dutta, D. Gaskell, J. W. Martin, K. D. Paschke, V. Tvaskis et al., Precision Electron-Beam Polarimetry at $1 \mathrm{GeV}$ using Diamond Microstrip Detectors, Phys. Rev. X 6, 011013 (2016).

[93] T. J. Gay and F. B. Dunning, Mott electron polarimetry, Rev. Sci. Instrum. 63, 1635 (1992).

[94] D. Gaskell, D. G. Meekins, and C. Yan, New methods for precision Møller polarimetry? Eur. Phys. J. A 32, 561 (2007). 